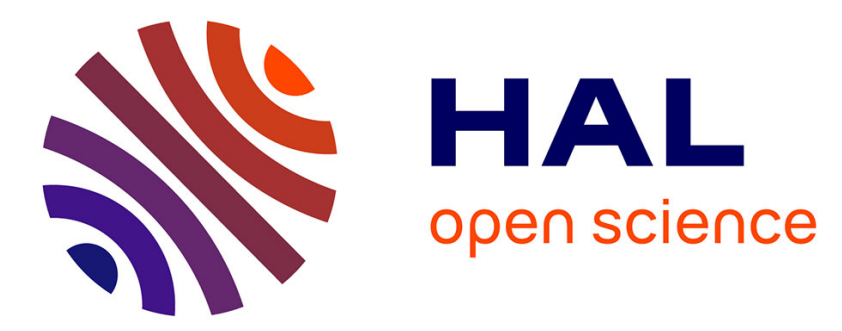

\title{
Defect pressure, formation volume, and temperature dependence of formation properties of point defects in ionic solids
}

R. Bauer, R. Leutz

\section{- To cite this version:}

R. Bauer, R. Leutz. Defect pressure, formation volume, and temperature dependence of formation properties of point defects in ionic solids. Journal de Physique Colloques, 1980, 41 (C6), pp.C6-516C6-519. 10.1051/jphyscol:19806135 . jpa-00220044

\section{HAL Id: jpa-00220044 https://hal.science/jpa-00220044}

Submitted on 1 Jan 1980

HAL is a multi-disciplinary open access archive for the deposit and dissemination of scientific research documents, whether they are published or not. The documents may come from teaching and research institutions in France or abroad, or from public or private research centers.
L'archive ouverte pluridisciplinaire HAL, est destinée au dépôt et à la diffusion de documents scientifiques de niveau recherche, publiés ou non, émanant des établissements d'enseignement et de recherche français ou étrangers, des laboratoires publics ou privés. 


\title{
Defect pressure, formation volume, and temperature dependence of formation properties of point defects in ionic solids
}

\author{
R. Bauer and R. K. Leutz \\ Institut für Theoretische und Angewandte Physik der Universität und \\ Institut fur Physik am Max-Planck-Institut für Metallforschung, \\ Pfaffenwaldring 57, D-7000 Stuttgart-80, FRG
}

\begin{abstract}
Résumé. - La conception de la pression locale d'un défaut est développée et appliquée à la détermination de la relaxation élastique autour d'un défaut ponctuel. La méthode est supérieure à celle des forces Kanzaki pour le trattement de l'interaction coulombienne et des interactions polyatomiques. On donne les sommes de réseau pour l'interaction coulombienne et on dérive le volume de relaxation des défauts Schottky dans $\mathrm{NaCl}$ et $\mathrm{KCl}$. L'utilisation des cubes Evjen neutres au lieu des domaines Mott-Littleton chargés est trouvée indispensable pour un traitement atomistique complet et satisfaisant de défauts chargés dans les solides ioniques. Dans les halogénures alcalins ainsi que dans les halogénures de l'argent la dépendance de température du volume et de l'enthalpie de formation des défauts en équilibre est comprise selon la dépendance de température des modules de cisaillement. Les anomalies à températures élevées du volume de formation des défauts, de la diffusion à traceur et de la conductivité ionique des halogénures de l'argent sont ramenées à une propriété intrinsèque du défaut Frenkel causé par une dépendance' de température exceptionnelle des propriétés élastiques du cristal parfait.
\end{abstract}

\begin{abstract}
The concept of local defect pressure is developed and is applied to determine the elastic relaxation about point defects in ionic solids. The method is superior to that of Kanzaki forces for treatment of Coulomb and many-atom interactions. The lattice sums for the Coulomb interaction and the relaxation volume of Schottky defects in $\mathrm{NaCl}$ and $\mathrm{KCl}$ in the static limit are given; the use of neutral Evjen cubes rather than charged MottLittleton regions is found unavoidable for a complete and satisfactory atomistic treatment of charged defects in ionic solids. In both alkali and silver halides, the temperature dependence of the volume and the enthalpy of formation of equilibrium defects is understood in terms of the temperature dependence of the shear moduli. The high-temperature anomalies of defect formation volume, tracer diffusion, and ionic conductivity of the silver halides is traced to an intrinsic property of the Frenkel defect caused by an exceptional temperature dependence of elastic propertue of bi perfect cryal
\end{abstract}

1. Introduction. - The understanding of elastic relaxation volumes associated with the formation of atomic defects in ionic solids is a long-standing problem. $\mathrm{Me}^{++}$impurities in $\mathrm{KCl}$ [1] have small negative relaxation volumes $\Delta V=-0.1 V_{c}$, $\left(V_{c}=V_{\text {cell }}\right)$, while for the Schottky defect in $\mathrm{NaCl}$, $\mathrm{KCl}$ and $\mathrm{KBr}$ there is ample experimental evidence [2-9] for a positive outward relaxation. Radiation damage data for $\mathrm{MgO}[10,11]$, on the other hand, indicate a contraction about the anion vacancy. Theoretical analysis, though, in the framework of an extended Mott-Littleton (ML) scheme [12, 13] using the method of Kanzaki forces [14. 15] yields strongly negative relaxations $[13,16,17]$ with $\Delta V=-0.7 V_{\mathrm{c}}$ for Schottky defects in both alkali halides [16] and $\mathrm{MgO}$ [17] caused by the dielectric body force. Flynn [19] claimed that the volume electrostriction of the polarized dielectric tending to increase the volume of the material should cause a positive relaxation volume.

Another group of open questions is concerned with the temperature dependence of defect properties which is possibly responsible for the curvature of $\log (\sigma T)$ versus $1 / T$ conductivity Arrhenius plots in the intrinsic region. The small and steady curvature found for alkali halides (AX), e.g. for $\mathrm{NaCl}$ [20], has tentatively been explained by other types of intrinsic point defects contributing slightly at high temperatures [21, 22], assuming that the Schottky defect formation parameters determined to within $1 \%$ are temperature independent. For the silver halides with $\mathrm{NaCl}$ structure $(\mathrm{AgX})$, the strong excess effects observed above $550 \mathrm{~K}$ in the defect formation volume [23], the ionic conductivity [24-26], and the diffusivity $[27,28]$ have been shown $[27,28]$ to be caused by a concentration enhancement of Frenkel [29$31]$ defects rather than by the onset of additional [23, 25] mechanisms. But the concentration enhancement can be explained neither by the Debye-HückelLidiard (DHL) theory and its extensions [26, 27] nor by the temperature dependence of the dielectric relaxation [27] yielding curves of the wrong shape, 
and there seems to be agreement that efforts using the mean field approach [32] are not likely to give a satisfactory explanation, either.

2. The macroscopic model. - We consider the continuum model of the vacancy which can be derived from the macroscopic hollow-sphere problem, figure 1, with outer pressure $p$, inner pressure and radius $p_{1}$ and $R_{1}$, and a charge $q$ at the center. The material is described by its dielectric constant $\varepsilon$, its second order elastic constants $c_{i j}$ or $\lambda, \mu$, and their respective dependence on $p$ and temperature $T$. The dielectric solution neglecting elastic strain and electrostriction has been used as a model for the vacancy in ionic solids by Schottky [33] and Jost [34]; the model has been substantiated by the dipolar lattice summations of ML [12].

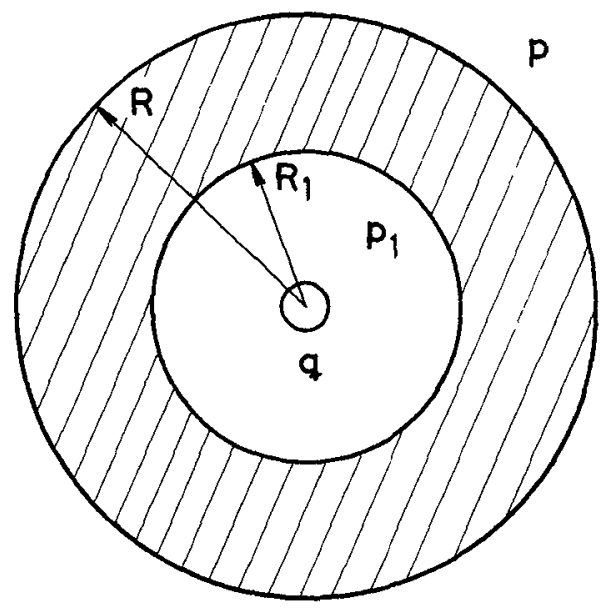

Fig. 1. - Hollow-sphere problem.

For the complete solution three additional displacement fields $\mathbf{s}(\mathbf{r})$ are needed $\left(\mathbf{r}=\left(x_{\mathbf{i}}\right) ; r^{2}=\mathbf{r} \cdot \mathbf{r}=x_{\mathbf{i}} x_{\mathbf{i}}\right)$, the homogeneous displacement

$$
\mathbf{s}^{h}(\mathbf{r})=C^{h} \mathbf{r}
$$

with homogeneous dilatation

$$
\Delta V^{h} / V=\eta_{i i}^{h}=3 C^{h}=\text { constant }(\mathbf{r})
$$

where $\eta_{\mathrm{ij}}$ is the strain tensor; the local displacement

$$
\mathbf{s}^{l}(\mathbf{r})=C^{l} \mathbf{r} / r^{3}
$$

with local singular dilatation at $\mathbf{r}=0$ :

$$
\eta_{\mathrm{ij}}^{l}=4 \pi C^{l} \delta(\mathbf{r}) ; \Delta V^{l}=4 \pi C^{l}=\operatorname{constant}(V) ;
$$

and

$$
\mathbf{s}^{r}(\mathbf{r})=-C^{r} \mathbf{r} / \mathbf{r}^{4}
$$

with $r$ dependent dilatation $\eta_{\mathrm{i}}^{r}=C^{r} / r^{4}$ concentrated near $\mathbf{r}=0$.

A more detailed derivation of the main results presented here will be given elsewhere (R. Bauer and R. K. Leutz, to be submitted to Solid State
Commun.). The basic quantity determining the elastic solution is the effective bulk modulus of the spherically concave medium $\tilde{K}$ [35]

$$
\tilde{K}=\mu 4 / 3 \text { or } \tilde{K} \simeq \bar{G} 4 / 3
$$

given by the shear modulus $\mu$ for isotropic media or by the Voigt mean shear modulus

$$
\bar{G}=\left(c_{11}-c_{12}+3 c_{44}\right) / 5
$$

for elastically anisotropic media; it is orthogonal to the bulk modulus $K=\left(c_{11}+2 c_{12}\right) / 3$. The elastic equilibrium and boundary conditions lead to the total outer volume change of the sphere, for $R_{1} / R \ll 1$ :

$$
\begin{array}{rlrl}
\Delta V^{\text {tol }} & =\Delta V^{l}+\Delta V^{h}=\gamma \Delta V^{l} ; & \gamma & =1+\tilde{K} / K \\
\Delta V^{l} & =\left(p_{1}-p+\sigma_{1}^{\text {diel }}\right) V_{1} / \tilde{K} ; & V_{1}=R_{1}^{3} 4 \pi / 3 .
\end{array}
$$

Here $\gamma$ is the Eshelby factor [36], and

$$
\sigma_{i}^{\text {diel }}=-f_{\varepsilon} q^{2} /\left(8 \pi R_{1}^{4}\right)
$$

is the dielectric boundary stress including the dielectric body force considered also in $[13,16-18]$ as well as the quasi-forces due to the incompatible [37] electrostrictive strain; the numerical factor $f_{\varepsilon}$ depends mainly on $\varepsilon$ and on its pressure derivative [38] describing the volume electrostriction which reduces $\sigma_{1}^{\text {diel }}$ by $30 \%$ but does not reverse its negative sign. This confirms Flynn's proposal [19] qualitatively but not quantitatively. The low temperature data of $[38,39]$ yield $f_{\varepsilon} \simeq 1.5$ for $\mathrm{NaCl}$ and $\mathrm{KCl}$.

3. The local defect pressure. - The continuum model of the vacancy is obtained by choosing the atomic Wigner-Seitz volume $V_{\kappa}$ for $V_{1}$, where the ion at site $\varkappa$ with valency $q_{\kappa}$ has been removed to form the vacancy and $q=-q_{\kappa}$ is the effective charge of the vacancy. Then $p_{1}$ is to be replaced by the local defect pressure of the vacancy which we define as

$$
p_{-\kappa \mid 0}^{l}=-\partial F_{-\kappa}^{\mathrm{tot}} /\left.\partial V_{\kappa}^{l}\right|_{T \mid \Delta V_{\kappa}^{L}=0} .
$$

Here $F_{-\kappa}^{\text {tot }}=F^{\text {tot }}+F_{\kappa}^{\text {sep }}$ is the total cohesive free energy of the crystal with vacant site $\varkappa$ and $F_{\kappa}^{\text {sep }}$ is the separation free energy of ion $\varkappa$. The local derivative (7) is a counterpart of the equilibrium condition

$$
p=p^{h}=-\partial F_{\kappa}^{\mathrm{coh}} /\left.\partial V_{\kappa}^{h}\right|_{T}
$$

which comprises a homogeneous derivative of the cohesive free energy per ion $\varkappa$. The various derivatives of $F$ are best expressed by pressure lattice sums $\pi_{\kappa}^{l, t}$ and $\pi_{\kappa}^{h, t}$ for an interaction potential of type $t$. For nearest-neighbour (nn) interactions $\pi^{l, t}=\pi^{h, t}$ while for long range interactions like the Coulomb potential the local and homogeneous derivatives differ strongly. With the important relation

$$
F^{\mathrm{sep},(m)}=-m F^{\mathrm{coh},(m)}[40]
$$


for many-atom potentials of order $m$, one gets for short range interactions by (8)

$p_{-\kappa \mid 0}^{l}=c\left(-2 p+\sum_{m=3}^{\hat{m}}(m-2) \partial F_{\kappa}^{\mathrm{coh},(m)} / \partial V_{\kappa}^{h}\right)$,

which shows that many-atom potentials give particular contributions to the defect pressure. This is important for the $\mathrm{AgX}$ and for oxides and has not been considered for $\mathrm{MgO}[17,18,41,42]$, where also the particular many-atom contributions to the formation energy $[40$, 43, 44] are neglected; these contributions are not accessible in a shell model [45] approach [17]. With a nn two-atom interaction for the $\mathrm{AX}$, we obtain for the $\mathrm{NaCl}$ structure in the static approximation, by (8) :

$$
p_{-\kappa \mid 0}^{l}=\left(\pi_{\mathrm{Mad}}^{l}-\pi_{\mathrm{Mad}}^{h}\right) e_{0}^{2} /\left(4 \pi r_{0}^{4}\right)-6 p / 4 \pi
$$

The pressure lattice sums related to the nn distance $r_{0}$ are $\pi_{\mathrm{Mad}}^{l}=3.863164, \quad \pi_{\mathrm{Mad}}^{h}=\alpha_{\mathrm{Mad}}=1.74756 . \quad \pi^{l}$ depends on the elastic anisotropy and is here given for the isotropic field (2). $\pi_{\text {Mad }}^{h}$ is equal to the energy Madelung sum $\alpha_{\text {Mad }}$ since with a homogeneous dilatation the crystal structure remains unaltered

For the Schottky defect, we take for $R_{1}$ in $\sigma_{1}^{\text {diel }}$ in (6) the mean polarization radius for which $\mathrm{ML}$ [12] have obtained $0.76 r_{0}$ and $0.73 r_{0}$ for $\mathrm{NaCl}$ and $\mathrm{KCl}$, respectively. Then $\sigma_{1}^{\text {diel }}$ and $p_{-\kappa \mid 0}^{l}$ cancel approximately in (6) and $\Delta V^{\text {tot }}=\Delta V_{\mathrm{S}}^{\mathrm{f}}$ is nearly zero in the static approximation. The thermal contribution to $p_{-\kappa \mid 0}^{l}$ increasing linearly with $T$ at high temperatures contributes to the positive relaxation volume found experimentally [2-9]. Another effect not yet considered is the elastic anisotropy and its strong temperature dependence for $\mathrm{AX}$ and $\mathrm{AgX}$ which affects the $\pi^{l, t}$. The explicite dependence of $(6)$ and $(10)$ on $p$ indicates a marked decrease of $\Delta V^{\text {tot }}$ with pressure which is seen in the data of [4-6]. For $\mathrm{Me}^{++}$impurities, $p_{\mathrm{Me}}^{l, \mathrm{C}}+p_{-\kappa \mid 0}^{l, \mathrm{C}}$ contracts the lattice and causes together with the effect of the associated vacancy a small negative relaxation in agreement with experiment [1].

4. Temperature dependences. - If we unite all local pressure contributions noted above in $p_{\mathrm{D}}^{l}(T)$, we may write

$$
\Delta V_{\mathrm{D}}^{\mathrm{f}}=\gamma\left(p_{\mathrm{D}}^{l}-p\right) V_{\mathrm{c}} / \tilde{K}
$$

where D stands for the type of defect, Schottky or Frenkel. Integrating the isothermal work done by $p_{\mathbf{D}}^{l}$ on the concave medium, we obtain the elastic free enthalpy of relaxation of the defect,

$$
\Delta G_{\mathrm{D}}^{\mathrm{f}}=-\frac{1}{2}\left(p_{\mathrm{D}}^{l}-p\right)^{2} V_{\mathrm{c}} / \widetilde{K}+p \Delta V_{\mathrm{D}}^{\mathrm{f}}
$$

and an entropy contribution $\Delta S_{\mathrm{D}}^{f}=f(T) / \tilde{K}^{2}+S_{\mathrm{D}}^{h}$. The temperature dependence of $\widetilde{K}$ is shown in figure 2 for $\mathrm{NaCl}[39,46]$ which is typical for $\mathrm{AX}$, and for $\mathrm{AgBr}[47]$ representing the $\mathrm{AgX}$. The intrinsic conduc-

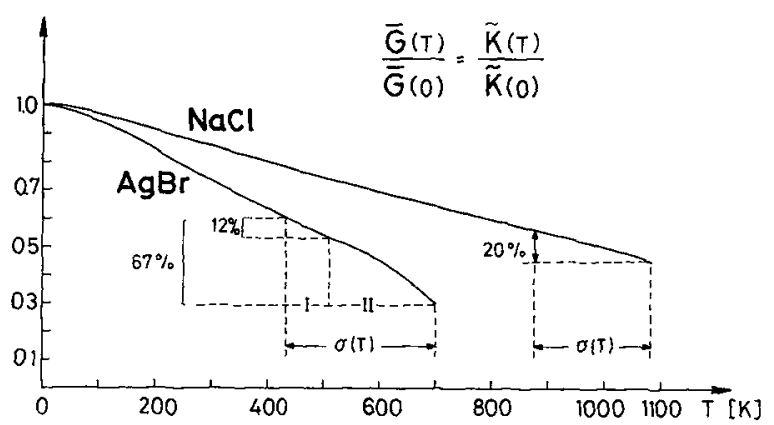

F1g. 2. - Normalized temperature dependence of the Voigt mean shear modulus $\bar{G}$ and of the effective bulk modulus of the spherically concave medium $\tilde{K}$ for $\mathrm{NaCl}$ [Landolt-Börnstein, 1966] and for $\mathrm{AgBr}$ [Tannhauser et al., 1956].

tivity regions of $[20]$ and $[26,27]$ are marked; region I contains the data assumed to be normal by Friauf et al. [26, 27, 32] and used to fit defect parameters while II is the range considered as anomalous $[26-28,32]$. Since $\widetilde{K}$ enters the denominator of (11) and (12), a linear decrease of $\widetilde{K}$ with $T$ leads to a non-linear decrease of $\Delta G_{D}^{f}$ causing a temperature dependence of formation enthalpies and entropies. Qualitatively, AX and AgX behave similarly; there are yet marked quantitative differences in both the drop of $\widetilde{K}$ from $0 \mathrm{~K}$ to $T_{\mathrm{M}}$ and the intrinsic conductivity range. If for $\operatorname{AgBr} \Delta V_{\mathrm{F}}^{\mathrm{f}}$ is taken from [23] and $p_{\mathrm{D}}^{l}(T)$ kept constant, $\Delta G_{\mathrm{F}}^{\mathrm{f}}$ decreases by $140 \mathrm{meV}$ between $500 \mathrm{~K}$ and $700 \mathrm{~K}$ and the shape of the curve fits well the conductivity anomaly $[26,27]$. A linear increase of $p_{\mathrm{D}}^{l}(T)$ dispenses even in region $\mathrm{I}$ with part of the DHL corrections which are not appropriate [48] for the high defect concentrations in $\mathrm{AgBr}$.

5. Discussion. - In the perfect neutral crystal every ionic charge $q_{k}$ is surrounded by its compensating charge $-q_{\kappa}$ visualized by neutral Evjen cubes [49]. The latter has a finite self-energy $\alpha_{\text {Mad }} q_{k}^{2} / 2 r_{0}$ depending on the positions of the neighbouring ions. In previous treatments of the relaxation, e.g. $[13,16-18$, $21,41,42]$, this charge and the local derivative of its self-energy are not included while they are contained in (7), (10); the ML method using highly charged regions $I$ and II is compelled to avoid consideration of net charges in I and II except the defect charge $q$ and is thus unable to keep trace of the compensating charge. Kanzaki forces being virtual quantities are useful to represent the elastic strength of a defect once the relaxation has been calculated, whereas the real defect pressure serves to find the relaxation itself. An atomistic treatment has to use neutral Evjen cubes rather than charged ML spheres and the elastic strength determining the macroscopic elastic displacement field (2) has to be treated as an independent variable in the energy minimization. Both has been achieved in [50] where a nearly zero elastic relaxation is found for $\mathrm{AX}$ in the static limit. This is now corroborated by the present continuum model. 
The wrong elastic relaxation in previous work affects strongly the migration energy of vacancies; the Schottky formation enthalpy of AX increases due to the elastic contribution by $6 \%$ within the intrinsic range. In fitting conductivity data, inclusion of temperature dependent formation parameters is vital for both $\mathrm{AX}$ and $\mathrm{AgX}$; constant parameters given to within $1 \%$ have little physical significance.

The model $G_{\mathrm{D}}^{\mathrm{f}}=C_{\mathrm{D}} K V_{\mathrm{c}}$ for the Gibbs free enthalpy of formation of defects $D$ has recently been discussed extensively by Varotsos et al., e.g. [51-53]. The rigorous expressions $(11,12)$ show that $-1 / \widetilde{K}(T)$ is important rather than the bulk modulus $K(T)$; since the temperature dependences of both are similar with each other for many materials the model accidentally works well. It is to be noted that the elastic stored energy of defects considered usually [54-57] and being positive is only part of the total negative elastic energy (12).

Our result for the interpretation of the $\mathrm{AgX}$ anomalies confirms the early proposal of Jost [58] discussed by Schmalzried [59] and that of Teltow and coworkers [60-62] which connect the formation volume with the formation free energy and assume an intrinsic temperature dependence of Frenkel defect properties. Likewise it confirms the conclusions of Slifkin [28] and Friauf [27].

Acknowledgements. - This work has been sponsored in part by the DFG. We thank Dr. Paus and Dr. Schwan for help with the preparation of the paper.

References

[1] Pick, H. and Weber, H., Z. Phys. 128 (1950) 409.

[2] Biermann, W., Z. Phys. Chem. Frankfurt 25 (1960) 90; 253.

[3] Beyeler, M. and Lazarus, D., Z. Naturf. 26a (1971) 291.

[4] Lazarus, D., Yoon, D. N. and JefFery, R. N., Z. Naturf. $26 a$ (1971) 56.

[5] Yoon, D. N. and Lazarus, D., Phys. Rev B 5 (1972) 4935.

[6] Martin, G., Lazarus, D. and Mitchell, J. L., Phys. Rev. B 8 (1973) 1726.

[7] Lüty, F., Costa Riberro, S., Mascarenhas, S. and SVerzut, V., Phys. Rev. 168 (1968) 1080.

[8] Lohstöter, H., Spatt, H. and Peisl, H., Phys. Rev. Letters 29 (1972) 224

[9] Spalt, H., Lohstöter, H. and Peisl, H., Phys. stat sol. (b) 56 (1973) 469

[10] Hickman, B. S. and Walker, D. G., Phil. Mag. 11 (1965) 101

[11] Henderson, B., Bowen, D. H., Briggs, A. and King, R. D., J. Phys. C: Solid St. Phys. 4 (1971) 1496.

[12] Mott, N. F. and Littleton, M. J., Trans. Farad. Soc. 34 (1938) 485.

[13] Hardy, J. R. and Lidiard, A. B., Phil. Mag. 15 (1967) 825.

[14] Kanzaki, H., J. Phys. Chem. Sol. 2 (1957) 24, 37.

[15] Hardy, J. R., J. Phys. Chem. Sol. 15 (1960) 39; 29 (1968) 2009.

[16] FaUX, I. D. and Lipiard, A B., Z. Naturf. 26a (1971) 62.

[17] Catlow, C. R. A., Faux, I. D. and Norgett, M J., J. Phys. C : Solid St. Phys. 9 (1976) 419.

[18] Puls, M. P., Woo, C. H. and Norgett, M. J., Phll. Mag. 36 (1977) 1457

[19] FLYNN, C. P., Z. Naturf. 26a (1971) 99.

[20] Allnatt, A. R., Pantelis, P. and Sime, S. J., J. Phys. C: Solid St. Phys. 4 (1971) 1778

[21] Catlow, C. R. A., Diller, K. M., Norgett, M. J., Corish, J., PARKer, B. M. C. and JACOBS, P. M. W., Phys. Rev. B 18 (1978) 2739

[2] licoBs, P. M. W. J Phwiqu Collod 41 (1980) C6-72, ( 6.207)

[23] KURNKK, S. W, J. Chem Phys. 20 (1952) 218.

[24] Weber, M. D. and Friauf, R. J., J. Phys. Chem. Sol. 30 (1969) 407.

[25] CoRish, J. and JacoBs, P. M W., J. Phys. Chem. Sol. 33 (1972) $1799:$ Phls, stat. sol (b) 67 (1975) 263.

[26] Aboagye, J. K. and Friauf, R. J., Phys. Rev. B 11 (1975) 1654

[27] Friauf, R. J., J. Physique 38 (1977) 1077.

[28] Batra, A P. and Slifkin, L. M., Phys. Rev. B 12 (1975) 3473 J. Physique Colloque 37 (1976) C7-396.

[29] Fouchaux, R. F. and Simmons, R., Phys. Rev. 136 (1964) A 1664

[30] Berry, R. Ch., Phys, Rev. 82 (1951) 422; 331
[31] Strelkow, P G, Phls/h. Z. Sowlommom 12 (1937) 73.

[32] Friauf, R. J., J. Physique Colloq. 41 (1980) C6-97.

[33] Sсноттку, W., Z. physik. Chemie, Abt. B, 29 (1935) 335.

[34] Jost, W., Z. physik. Chemle, Abt. B, 32 (1936) 1.

[35] KRÖNER, E., Acta Met. 2 (1954) 302.

Nabarro, F. R. N., Proc. Roy. Soc. (London) A 175 (1940) 519.

[36] Eshel by, J. D., J. Appl. Phys. 25 (1954) 255.

[37] Kröner, E., $\mathcal{Z}$. angew. Physik VII (1955) 249

[38] Lowndes, R. P. and Martin, D. H., Proc. Roy. Soc. (London) A $316(1970) 351$.

[39] Haussühl, S., Z. Physik 159 (1960) 223.

[40] BAuer, R. and Leutz, R. K., Phys. Lett. A, to be published.

[41] Mackrodt, W. C. and Stewart, R. F., J. Phys. C 10 (1977) 1431.

[42] Stewart, R. F. and MaCkrodt, W. C., J. Physique Colloq. 37 (1976) C7-247.

[43] Foreman, A. J. E. and Lidiard, A. B., Phil. Mag. 8 (1963) 97.

[44] Losee, D. L. and Srmmons, R. O., Phys. Rev. 172 (1968) 934.

[45] Sangster, M. J. L., J. Phys. Chem. Sol. 34 (1973) 355.

[46] Landolt-Börnstein, New Series, Group III, Vol. 1 , Bechmann, R. and Hearmon, R. F. S. (Springer), 1966.

[47] TA\NHAuser, D. S., Bruner, L. J. and LAWSON, A. W Phys. Rev. 102 (1956) 1276.

[48] Fryer, G. M., Phil. Mag. 32 (1975) 173.

[49] Tosi, M. P., Solid State Physics 16 (1964) 1.

[50] LeUTZ, R. K., Thesis, Universität Stuttgart (1978).

[51] Varotsos, P., Phys. Rev. B 13 (1976) 938.

[52] Varotsos, P. and Alexopoulos, K., Phys. Rev, B 15 (1977) $4111 ;$ J. Phys. Chem. Sol. 38 (1977) 997.

[53] Varotsos, P., Ludwig, W. and Alexopoulos, K., Phys, Rev. B $18(1978) 2683$.

[54] Zener, C., J. Appl. Phys. 22 (1951) 372; Imperfections in Nearly Perfect Crvstals (Wiley, New York) 1952, pp. 289 316

[55] Seeger, A. and ManN, E., Z. Naturf. 14a (1959) 154.

[56] Schotrky, G., Seeger, A. and Schmid, G., Phys. stat. sol. 4 (1964) 439 .

[57] Holder, J. and Granato, A. V., Phvs. Rev. 182 (1969) 729.

[58] Jost, W. and NeHLep, G., Z. physik. Chem. Abt. A, 169 (1934) 129 ; Abt. B, 34 (1936) 348.

[59] Schmal.zRied, H., Z. Physik. Chem. Neue Folge 22 (1959) 199.

[60] Tel Tow, J., Ann. Physik (6) (Leipzig) 5 (1949) 63; 71.

[61] Enert, I. and Teltow, J., Ann. Physik (6) (Leipzig) 15 (1955) 268 .

[62] MÜl.I IR, P, Phys stat. sol. 12 (1965) 775, 21 (1967) 693. 